\title{
POLÍTICA AGRÍCOLA EN COSTA RICAY SU EFECTO SOBRE EL CAMPESINADO. ¿TENDRÁ LA EDUCACIÓN “ALGUNA VELA EN ESTE ENTIERRO”?
}

\section{M.Sc. Fernando Sáenz Segura*}

Política agrícola. campesinado y educación son tres temas que se ponen juntos en este documento, y se analizan dentro de un contexto particulamente difícil para los pequeños productores agropecuarios de Costa Rica. Aunque el término política agrícola se refiere a las formas en que el Gobiemo se propone objetivos y metas para el sector agropecuario, y los métodos para alcanzarlos, también la indefinición de política es una opción de políica en sí. Este documento hace un recuento de como se pasó de un modelo de desarrollo asistencialista a uno de libre mercado, en donde la inexistencia de una política agricola definida parece ser la noma. Esto ha sido particularmente grave para los pequeños productores campesinos de Costa Rica, a los cuales se les aplicaron las mismas políticas de sesgo agro-exportador que a los grandes productores, y sin una adecuada estrategia de preparación. A lo largo de veinte años los pequeños productores agropecuarios han sufrido en su mayoría exclusión económica y pobreza, situación que pone en peligro la existencia de tejido social en las zonas rurales El análisis se amplía hacia el papel asumido por la educación hasta el momento, y' cuáles han sido sus implicaciones en este proceso de cambio en el sector agropecuario.
Agrarian policy, rural population and education are three issues which are combined in this study and examined in a particularly difficult context for small farmers in Costa Rica. Although the term agrarian policy refers to the ways in which the govermment proposes objectives and goals for the farm sector: and the methods to be used to reach them, the lack of defined policies is a political option in itself. This study describes the change from an aid-oriented development model to that of the free market. where the lack of a defined agrarian policy. appears to be the norm. This has been par. ticularly serious for the small farmers in Costa Rica, who were affected by the same agro-export bias policies as those applied to the large producers, with no adequate preparatory strategy. Most small farmers have suffered from economic exclusion and poverty for 20 years. This situation has endangered the existence of the social network in rural areas. The analysis is extended to cover the role assumed by education to the present time, and includes its implications in this process of change in the farm sector:

* Economista agrícola de la Universidad de Costa Rica, máster en Manejo de Recursos Naturales por el Centro Agronómico Tropical de Investigación y Enseñanza (CATIE), estudiante de doctorado en Economía Agrícola en la Universidad de Wageningen, Holanda. Participa en proyectos de investigación del Centro Internacional de Política Económica (CINPE) en asocio con la Universidad de Wageningen. También, ejerce la docencia. fsaenz@una.ac.cr 


\section{Introducción}

Me gustaría empezar esta introducción con un vehemente Sí: la educación sí tiene un papel muy importante en la reivindicación del campesino en Costa Rica y su aporte a la sociedad. Esta idea es particularmente importante de replantear en un país pequeño como Costa Rica, donde el sector agropecuario ya no tiene la importancia que tenía antes, donde el nuevo proceso de mundialización transformó sus relaciones con otros países y donde las ideas de campesino y cultura campesina son en la actualidad cada vez más extrañas y lejanas para mucha gente, especialmente en las áreas urbanas.

La idea de este artículo es relacionar tres aspectos que a simple vista parecen inconexos: política económica, campesinado y educación, los cuales tienen más bien un sentido integral e importante para el desarrollo de aquellas zonas del país de fuerte carácter agropecuario. Para desarrollar esta idea es importante definir primero qué es lo que llamamos "políica" en este documento, específicamente política agropecuaria y cuáles son las especificaciones de politica que se pueden encontrar en agricultura. Después es importante pensar que en agricultura hay campesinos y productores, los cuales no son lo mismo, ya que existen grandes diferencias entre ellos. Se sigue con un bre ve recuento de lo que ha sido la política agropecuaria en Costa Rica, qué efectos positivos y negativos tuvieron para estos diferentes tipos de productores y por qué se ha dado una situación desigual.

Este análisis se amplía también hacia el papel asumido por la educación hasta el momento y cuáles han sido sus implicaciones en este proceso de cambio en el sector agropecuario.

Finalmente, se propone una agenda de discusión, donde la educación está llamada a asumir un nuevo papel, más reivindicativo y fortalecedor del campesinado en Costa Rica, y menos exógeno a una realidad cada vez mas difícil para los pequeños productores agropecuarios.

\section{Política económica}

\subsection{Definnición}

Frank Ellis en su libro Politicas agricolas en paises en vias de desarrollo (1992) afirma que no existe una definición única del término "politica", pero en general entre economistas se piensa como políticas en todos aquellos métodos y metas por medio de los cuales un gobierno interviene en la economía, afectando variables como precios, tasas de interés, nivel de ingreso, etc. En general, se 
habla de "política" cuando se trata de la intervención del Estado en la economía y se habla de políticas cuando se trata de intervenciones específicas. Un par de definiciones sencillas son las siguientes:

"Política es el curso de acción que sigue el Gobierno en un aspecto específico de la economía, para lo cual se fija metas y escoge métodos para alcanzarlas" (Ellis, 1992).

O bien:

"Es el conjunto de actuaciones económicas realizadas por el Gobierno y otros órganos del Estado, para alcanzar ciertos objetivos macroeconómicos en el corto y en el largo plazo" (Díaz et al., 1995).

Sin embargo, como el mismo Ellis lo reconoce, la ausencia de intervención del Estado es una opción de política posible que, como se verá más adelante, se ha venido implementado cada vez más en países como Costa Rica.

\section{2) Niveles y tipos de política agrícola}

A partir de la anterior definición, se pueden identificar cuatro niveles de política:

1. Regional: se puede ver como el tipo de influencia que una economía dominante ejerce sobre otras economías más pequeñas. Ejemplos son: la política comercial que tiene Estados Unidos de América y la Comunidad Europea con zonas o regiones de América, tales como la Iniciativa de la Cuenca del Caribe (ICC), los Sistemas Generalizados de Preferencias (SGP), el Área de Libre Comercio de las Américas (ALCA), etc.

2. Nacional: es la directriz que ejerce el Gobierno de un país sobre un aspecto específico de la economía y que afecta al país como un todo. Ejemplos son: es la política monetaria, la política fiscal, la política de comercio exterior, etc. También aquí cabe la definición que hace el Gobierno en materia de educación, los objetivos que se plantean y los métodos para alcanzarlos.

3. Sectorial: se refiere más al efecto deseado por el Gobierno sobre un sector específico de la economía, como el agropecuario. Ejemplos son: es la política de subsidios, de inversión en infraestructura, política de crédito, tecnología, mercadeo, etc. 
4. Local: en este nivel se manejan decisiones referentes a una unidad geográfica en particular. Por ejemplo, las políticas de zonificación de cultivos para una región en particular, o bien, las políticas de manejo y protección de cuencas hidrográficas. Generalmente a este nivel se manejan dos criterios de división espacial: 1) el geográfico (de cuencas, o zonificación de cultivos), y 2) el político-administrativo (de cantonés o distritos).

Por lo general, la formulación y transmisión de políticas se manejan muy verticalmente en estos cuatro niveles; este hecho ha sido bastante criticado por investigadores, técnicos y organizaciones rurales de base. Así, por ejemplo, los niveles sectoriales y locales son generalmente meros receptores de la política económica que se decide en niveles nacionales (Banco Central, Ministerio de Hacienda, Comercio Exterior, etc.), e incluso, internacionales, cuando el país se supedita a lo que se decide en países de economías más grandes, a los cuales Costa Rica se vincula comercial, política y económicamente; o bien, a lo que se decide en grandes organismos internacionales que de igual forma tienen una gran influencia sobre los países en vías de desarrollo. Una situación muy similar sucede con la formulación de políticas en educación, las cuales se hacen de forma centralizada y verticalista, aplicando los mismos patrones y criterios tanto para las áreas urbanas como para las rurales.

Siguiendo a Ellis y relacionando política con lo que sucede en las unidades de producción agrícola, se puede enfocar el análisis más en términos de una relación de insumo-producto. De esta forma, hay tres categorías más comunes de intervención estatal en el sector agropecuario:

1. En el nivel de precios: de insumos y productos.

2. En el nivel de instituciones: mercadeo de la producción, acceso a tecnología e insumos, acceso a información.

3. En el nivel de cambio tecnológico: creación, difusión y adopción de tecnologías de producción.

Siendo más específico, se pueden definir los siguientes siete tipos de política sectorial:

1. Políticas de precios: afectan la estabilidad del precio recibido por el productor agropecuario.

2. Políticas de mercadeo: afectan la transferencia de productos desde la unidad de producción a los centros de consumo. 
3. Políticas de insumos: afectan los precios y el acceso de los insumos requeridos en la unidad de producción.

4. Políricas de crédito: se relacionan (no en exclusiva) con la provisión de capital de trabajo para financiar insumos variables en la unidad de producción.

5. Políticas de mecanización: estimulan o no la adopción de tecnologías mecánicas de producción o la inversión de activos fijos.

6. Politicas de reforma agraria: condicionan el acceso a la tierra como recurso para la producción agropecuaria y afectan la tenencia de la misma.

7. Políticas de investigación: tienen relación con la generación y extensión de nuevas tecnologías de producción y nuevos materiales genéticos, por lo que afectan fuertemente la productividad de la unidad de producción.

8. Politicas de irrigación: determinan la disponibilidad y uso del agua como insumo para la producción agropecuaria, por lo que afectan el área potencial de una actividad e implican inversiones públicas de gran escala.

Sin embargo, en esta distribución no se consideran políticas de educación agrícola, que integren programas de extensión y de formación, con procesos de educación formal e informal. Si bien es cierto que existen algunos esfuerzos puntuales en esta dirección, no se ha formulado de forma oficial una política de educación comunal que busque dos aspectos: 1) la transferencia generacional de conocimientos agrícolas, y 2) el fortalecimiento de un proceso de formación de jóvenes productores agropecuarios que sea sostenible en el tiempo.

\section{Estructura agraria en Costa Rica}

Antes de ver cómo ha evolucionado la política agropecuaria en Costa Rica, es importante tener claro quién es el "receptor" de esta política y qué tipos de diferencias puede haber dentro de este gremio. Estamos hablando del productor agropecuario. Cuando se habla de diferencias entre productores se trata de la estructura agraria, la cual es bastante diversa en Costa Rica. Así, unidades de producción altamente intensivas en el uso del capital y tecnología y más orientadas hacia el mercado exportador coexisten con haciendas ganaderas extensivas en el uso de la tierra y con pequeñas y medianas fincas o parcelas. Este último grupo es particularmente importante, porque es el más numeroso y diverso. En general está conformado por unidades de producción que son empresa y familia al mismo tiempo, por lo que tienen un carácter dual implícito (Ellis, 1988). En otras palabras, se trata de familias que producen bienes y servicios agrícolas y no agrícolas, que generalmente viven en la misma parcela o finca donde trabajan, que generan una economía familiar que se articula con la economía local y 
que toman decisiones de producción, consumo y reproducción de forma simultánea (Ellis, 1988).

Este grupo de pequeños y medianos productores es bastante diverso en cuanto a dotación de recursos e integración a los mercados. Así, pues, existen unidades con bastante tierra (especialmente en zonas remotas) y también existen parcelas de menos de cinco hectáreas. Además hay productores mas integrados al mercado, que producen productos tradicionales y no tradicionales (café, caña de azúcar. banano, plátano, hortalizas, plantas ornamentales, raíces y tubérculos) y otros más subsistentes, que prefieren garantizar su propia alimentación primero antes de pensar en comercializar alguno de sus productos.

Es importante tener claro también cuál es la diferencia entre un productor y un campesino. Mientras el primero simplemente se puede pensar como un ejecutor de una relación de insumos y productos, el segundo implica una cultura, una tradición, una herencia. Una persona con una finca, con recursos y un buen plan puede intentar producir cualquier producto de la tierra, pero decir que se es campesino implica una pertenencia a un modo de vida, a una filosofía y a un bagaje de conocimientos de tipo transgeneracional.

\section{Evolución de la política agropecuaria en Costa Rica}

\subsection{De 1950 a 1980: Impulso e inversión: Sustitución de importaciones}

Durante este periodo, la política agropecuaria se orientó a estimular el agro como motor de desarrollo. En esos años se promovió que el productor agropecuario, y muy en especial el sector campesino tradicional se refiere a productores de bajo ingreso, ubicados en antiguas zonas de frontera agrícola o en asentamientos creados por el Instituto de Desarrollo Agrario (IDA), quienes tradicionalmente producian granos básicos, maíz y ganado (a veces como subsistencia), utilizando niveles bajos de tecnología. Este sector se beneficiará con programas estatales tales como crédito subsidiado, precios de sustentación para granos básicos, subsidios a los insumos y otros factores de producción, y programas de investigación y extensión.

Estas políticas agrícolas tenían básicamente dos objetivos: 1) asegurar en una economia cerrada, todo el alimento que la naciente y urbana clase media iba a necesitar, y 2) apostar a que la economía del país iba a descansar principalmente en la producción de café, caña de azúcar, banano, granos básicos y ganaderia.

La implementación de estas políticas durante treinta años trajo modernidad al agro costarricense e implicaron inversión estatal en infraestructura de apoyo a la producción, crédito subsidiado, precios de sustentación y distribución 
de tierras. De esta forma, estas políticas contribuyeron a la estabilidad social y política del país por medio de un mayor desarrollo rural (Mora, 1994). Sin embargo, también en muchos casos, estas políticas terminaron beneficiando más a grandes productores con contactos políticos e influencias, que al amplio sector de pequeños y medianos productores campesinos para los cuales fueron pensadas (Cartín \& Piszk, 1980).

\subsection{0 en adelante: Crisis, ajuste estructural y apertura comercial}

El anterior modelo de desarrollo colapsó a principios de la década de los 80 , debido a su fuerte naturaleza intervencionista y proteccionista, que resultó en un crecimiento exagerado y poco eficiente del Estado. Otras razones importantes fueron:

- Presencia de mercados distorsionados.

- Dependencia de pocos productos de exportación.

- Fomento del consumo interno y desestímulo al ahorro.

- Fuerte proceso de endeudamiento externo.

- Corrupción y clientelismo político.

- Producción agrícola para consumo nacional dominada por pequeños y medianos productores, altamente dependientes de ayuda estatal, bajos niveles de organización, y con muy poca o nula vinculación con la agroindustria.

Esta situación devino en un deterioro general de la economía y en una disminución en el ingreso real, con el consecuente impacto en la calidad de vida del costarricense (SEPSA, 1997).

Después de 1980, la política agraria en Costa Rica cambió radicalmente, pasando de un esquema de protección y sustitución de importaciones, a uno de continua incorporación del sector agropecuario a la economía global de mercado, sin intervención estatal (Pomareda, 1996; SEPSA, 1997). En este sentido, se implementaron nuevas políticas como: la reducción de impuestos a las exportaciones e importaciones, devaluación monetaria, una mayor disponibilidad de financiamiento con tasas de interés atractivas para actividades agroexportadoras. De esta forma, se fortalecieron actividades tradicionales de exportación (café, caña de azúcar, y banano) y se promovió la adopción de nuevos productos no tradicionales de exportación como: piña, flores, plantas ornamentales, raíces y tubérculos y pescado fresco (González, 1994; Masís \& Rodríguez, 1994; Mora et al., 1994). Estas políticas de ajuste, fuertemente orientadas a la exportación, 
permitieron un mayor flujo de recursos financieros que fueron principalmente invertidos en la agricultura no tradicional, y promovieron un mayor cambio tecnológico, lo que permitió aumentos en la productividad. Adicionalmente se atrajo inversión extranjera, en especial en la producción de flores, ornamentales y follajes, macadamia y cítricos (Kaimowitz, 1992, citado por Mora, 1994).

De acuerdo con lo anterior. las políticas seguidas tuvieron como objetivo aumentar la productividad y la competitividad del sector agropecuario, con el fin de generar también mayores ingresos en la mano de obra empleada. Así, se esperaba un efecto positivo sobre el bienestar de la población rural y una mayor equidad social.

En general, la política sectorial agropecuaria ha variado muy poco desde entonces, sólo se ha agregado la creación del Programa de Reconversión Productiva (aprobado en diciembre de 1997 por la Asamblea Legislativa) y se ha promovido de algún modo una mayor participación campesina y una mayor conciencia sobre la conservación ambiental.

\section{Problemática: ¿A quiénes afectó negativamente el ajuste y cómo?}

Las políticas de ajuste afectaron en mayor parte al sector tradicional campesino, quienes enfrentaron súbitamente altos costos de producción (altos precios por insumos importados) y bajos precios por su producción tradicional (Mora $e t$ al. 1994). Esta situación se ha visto en particular agravada por la constante devaluación del colón y por la equiparación del valor de la producción nacional (en especial granos básicos), con precios internacionales fuertemente distorsionados por subsidios otorgados en países más fuertes en relación con lo económico.

Adicionalmente, las políticas de ajuste agravaron en frma indirecta el deterioro de la calidad de los recursos naturales que se utilizan en agricultura (suelo. agua y bosque) (Kruseman et al., 1994). Sin entrar a analizar el abuso en el uso de agroquímicos que hay en Costa Rica, los cultivos comercialmente más atractivos presentan paquetes tecnológicos muy intensivos en el uso de los mismos. Debido a falta de recursos económicos para trabajar y de más investigación, los pequeños productores enfrentaron (y enfrentan) el dilema de precios inestables por su producción, con alzas crecientes en los precios de los agroquímicos (la mayoría son importados). Por lo general, los productores más pobres sustituirán las fertilizaciones que no puedan pagar con extracción de nutrientes del suelo, causando el deterioro del mismo. También, habrá tumba del bosque de sus parcelas en busca de suelo fresco, con una mejor cantidad de nutrientes. Otro ejemplo es el de los ganaderos, mientras no haya mejores estimulos para la producción e investigación en sistemas viables de intensificación, la ganadería de carne en 
Costa Rica seguirá siendo extensiva en tierra y de baja productividad (de acuerdo con Mora et al. (1994), el 50\% de la tierra de las fincas en Costa Rica está usado como pastura).

Así, muchos pequeños productores sufrieron y todavía sufren de exclusión económica y pobreza entendida como una disminución a los mercados de insumos y productos (Mora et al., 1994). Adicionalmente a esto, durante los últimos veinte años se ha venido arraigando la idea de que la agricultura tiene que ser pobre, subdesarrollada, altamente demandante de esfuerzo físico y mal pagada. Lo preocupante de esta situción es que los hijos de los agricultores de la actualidad cada vez sienten menos estímulos para seguir los pasos de sus progenitores. Si se pone atención a los mensajes del Gobierno, sumados a lo que el medio dominante promueve (por medio de televisión, radio, etc.), los hijos de los agricultores querrán cada vez más buscar su futuro en otras actividades, e incluso, lejos de sus zonas de origen. De esta manera, se pone en peligro la construcción de un tejido social en las zonas rurales y se fomenta la inmigración hacia zonas urbanas.

\section{Causas de esta problemática}

Si repasamos lo que se ha mensionado antes, tenemos que la problemática reside básicamente en que las políticas de ajuste en Costa Rica se aplicaron por igual de forma homogénea, sin tomar en cuenta que la estructura agraria es heterogénea y

\section{Cuadro 1}

\section{La doble realidad que el ajuste ignoró del Sector Agropecuario (SA) en Costa Rica}

\begin{tabular}{|c|c|}
\hline $\begin{array}{l}\text { Diferencias entre } \\
\text { unidades de prod }\end{array}$ & Factores externos a la finca \\
\hline $\begin{array}{l}\text { 1. Dotación de recursos. } \\
\text { 2. Disponibilidad y demandade mano de obra. } \\
\text { 3. Acceso relativo a capital de trabajo. } \\
\text { 4. Nivel tecnológico. } \\
\text { 5. Integración al mercado. } \\
\text { 6. Escala de producción. } \\
\text { 7. Acceso a información, lo que puede re- } \\
\text { sultar en malas interpretaciones de las se- } \\
\text { ñales del mercado. } \\
\text { 8. Objetivos y comportamientos del productor. } \\
\text { 9. Uso de instituciones de mercado alternati- } \\
\text { vas que no han sido muy bien estudiadas. }\end{array}$ & $\begin{array}{l}\text { 1. Alta dependencia de insumos importados } \\
\text { y de capital de trabajo. } \\
\text { 2. Falta de una infraestructura apropiada para } \\
\text { mercadear sus productos. } \\
\text { 3. Mercados de insumos y productos frag- } \\
\text { mentados imperfectos, o inexistentes. } \\
\text { 4. Limitado grado de desarrollo institucio- } \\
\text { nal y organizacional. } \\
\text { 5. Predominancia del cálculo político sobre el cri- } \\
\text { terio técnico, a la hora de establecer decisiones } \\
\text { en el SA (distribución de tierras, por ejemplo). } \\
\text { 6. Fuertes recortes en programas de investigación } \\
\text { y extensión, con efectos negativos en acceso a } \\
\text { tecnologías y fuentes de información. }\end{array}$ \\
\hline
\end{tabular}


Lo preocupante de esta situación es que los hijos de los agricultores de la actualidad cada vez sienten menos estímulos para seguir los pasos de sus progenitores. Si se pone atención a los mensajes del Gobierno, sumados a lo que el medio dominante promueve (por medio de televisión, radio, etc.), los hijos de los agricultores querrán cada vez más buscar su futuro en otras actividades, e incluso, lejos de sus zonas de origen. De esta manera, se pone en peligro la construcción de un tejido social en las zonas rurales y se fomenta la inmigración hacia zonas urbanas. que el entorno económico en que se desarrollan las fincas no es tan homogéneo ni tan perfecto como se supuso. El siguiente cuadro resume esta doble problemática.

Las diferencias entre fincas o unidades de producción hacen que los productores tengan diferentes costos a la hora de transar en los mercados (diferentes costos de transacción), por lo que enfrentan también diferentes precios y diferentes pcrcepcioncs dc riesgo (Ellis, 1988; Sadoulet \& de Janvry, 1995).

Adicionalmente. los siguientes factores contribuyen a que la situación sea más difícil para los más pequeños del sector agropecuario:

- Alta dependencia de intermediarios tanto el que compra en finca, como el que vende directamente al consumidor (supermercados incluidos) para comercializar, con muy poca o ninguna información sobre los márgenes de comercialización que se manejan en agricultura. De hecho, Kaimowiitz (1992) señala que los pequeños productores aportan cerca del $35 \%$ del total de exportaciones no tradicionales de Centroamérica, lo que representaba cerca de US $\$ 100$ millones en el momento del estudio. Sin embargo. sólo reciben US\$ 30640 millones, quedándose el resto en manos de procesadores, exportadores y transportistas. En Costa Rica, por ejemplo. no existe un sistema de control y monitoreo de los márgenes de comercialización que manejan las grandes cadenas de supermercados.

- El desmantelamiento casi total de la investigación agropecuaria en el país. lo que ha dcjado en muchos casos el uso de variedades agotadas, el abuso de agroquímicos y la persistencia de bajos niveles tecnológicos en muchas unidades de producción.

- La aplicación de generosos subsidios a la producción agrícola por parte de países económicamente fuertes. Sólo en Estados Unidos se invirtieron USS 92.000 millones en los últimos cinco años y en la actualidad se da un debate 
para un nuevo programa de dicz años por unos US\$ 170.000 millones. Dc hecho, en Estados Unidos ya se promulgó la ley Farm Bill, que implica destinar US\$ 300.000 millones para respaldar la agricultura de ese país. y la cual ya está anunciada como fuera de toda ncgociación en un eventual Tratado de Libre Comercio entre Centro América y Estados Unidos (Lal Nación, 7 de octubre de 2002, página 20 A).

- La carencia de una estratcgia nacional de largo plazo que desarrolle y modernice al sector, incluyendo y no excluyendo a todos sus actores. Las últimas dos administraciones (Figueres Olsen y Rodrígucz Echeverría) se caracterizaron por cambiar de ministro de agricultura casi cada año, lo que revela una falta real de interés en cl futuro del sector y la ausencia de un nortc definido.

- La implementación reciente por parte de Costa Rica de una política comercial miope, más empecinada en firmar tratados de libre comercio a diestra y siniestra, que en analizar los efectos y cvolución de los mismos y establecer negociaciones para corregir defectos.

- La carencia de un censo agropecuario (el último fue en 1984) que pcrmita tener un diagnóstico real y complcto de la situación del sector en el país, y que oriente una cstrategia de trabajo de largo plazo.

- Políticas de educación mayoritariamente desvinculas de la antcrior rcalidad. que asume que los problemas y realidades de un hogar campesino son iguales a las de un hogar urbano.

\section{Conclusiones}

1. El cambio del esquema de sustitución de importaciones por un modelo de economía de libre mercado trajo oportunidades y amenazas para el sector agropecuario de Costa Rica. Por un lado. se aumentó cl potencial competitivo de muchas actividades (algunas novedosas), pero, por otro lado, hay ausencia de cstrategias apropiadas de coordinación e interrelación entre cl sector privado, el sector campesino y el Gobierno, con el fin de enfrentar los nuevos retos que la globalización plantea y sacarles provecho (SEPSA, 1999). Se entiende por globalización al proceso mundial que busca reducir costos de comunicación y transportc de bienes y servicios, la promoción del comercio de los mismos y el aumento de flujos de capital internacional e intercambios de tecnología (van den Noord, 1996).

2. La estructura agraria del país es heterogénea, lo que produce diferentes reaccioncs ante políticas agrarias homogéneas. Sin embargo, esta heterogeneidad se está perdicndo, pues el productor agropecuario se ha venido dividiendo cada vez más en dos grandes categorías distantes entre sí: los que fueron exitosos ante el cambio y los que no. Los primeros pasaron a engrosar el sector 
agroexportador, usan nuevas tecnologías de producción, han logrado integración vertical, acumulación de capital, y divcrsificación económica. Los scgundos se mantienen en las mismas actividades, usan bajo nivel tecnológico y están engrosando lentamente un sector rural cada vez más pobıe (Pomareda, 2000).

3. El paradigma cconómico de que el país debe producir sólo en lo que cs competitivo asume una rápida y automática intcgración de todos los diferentes tipos de productores al sector agrocxportador. Sin embargo, siguiendo estc paradigma no han podido superar los problemas existentes en cuanto a integración de mercados. innovación tecnológica, mercados de insumos y productos deficientes, mercados de tierra imperfectos y asimetrias de información (Gonzále\%, 1998).

4. La realidad agropecuaria del país indica que el paradigma de la agroexportación es un proceso más lento y complejo de lo que se esperaba. por lo que todavia hay un papcl importante del Estado y de la socicdad civil rural cn la promoción e implementación de nuevos servicios $\mathrm{c}$ instituciones entrc agentes económicos.

\section{Puntos propuestos para una agenda de discusión}

- Inversión en tecnología: producción de nuevas variedades e investigación en nuevos sistemas de producción que impliquen aumentos de productividad, menos dependencia de insumos importados y un máximo provecho de las potencialidades de nuestros agroecosistcmas.

- Inversión en sisfemas de información: se debe promover la creación de fuentes de información imparcial y precisa, de manera que sca asequible y de fácil comprensión para pequeños productorcs. Estos sistemas de información deberían de enfocarse cn: mercados y sus condiciones, oferta nacional de la producción, sistcmas de producción, tecnologías, precios de insumos y productos. De igual forma, los consumidores deben ser informados sobre que tan cicrto es que tienen precios bajos en los supermercados. Micntras no exista un sistema de monitoreo imparcial y continuo esta afirmación es inválida y sólo contribuirá a difundir desinformación entre consumidores.

- Valor agregado: la producción en finca y/o en las fases primarias de una agrocadena deben de orientarse hacia la incorporación cada vez mayor de un valor agregado al producto.

- Organización campesina: sigue siendo un factor muy importante y clave en el empoderamiento de agrocadenas, en la disminución de asimetrías de 
información, en la diversificación de mercados y en la reducción de márgenes de comercialización.

- Politica comercial: debe de incorporar los intereses de pequeños y medianos productores, los cuales sólo podrían verse representados por actores sociales transparentes. La política comercial tiene que dejar de ser sesgada hacia el simplismo de qué se importa y qué no, y ser más bien una promotora de desarrollo.

- Politica de educación diferenciada: esta debería de formularse diferente de la planteada para las zonas urbanas. al menos en los siguientes dos ejes:

1. Tomar en cuenta que los horarios y distancias en las zonas rurales son muy diferentes. Los niños son un componente de mano de obra para las economías campesinas, por lo que no se debe de asumir que ellos estarían dedicados exclusivamente a un rígido currículo de zona urbana (con rígidos horarios escolares), sino que en general aportan parte de su tiempo al trabajo en el campo. Los currícula escolares deberían de ser más acordes a esta realidad y dar herramientas más útiles que le sirvan al niño en su aporte a la economía familiar. Tal vez así, se disminuya la deserción en escuelas y especialmente en colegios, dando a los niños la idea de que la escuela está "sintonizada" con su realidad campesina, y que es más bien una fuente de información para resolver problemas comunes en las parcelas donde viven y trabajan.

2. Los currícula escolares deberían de promover más la formación de "campesinos más educados", no solamente como productores sino como los pequeños empresarios que deberían de ser. Si bien es cierto que esta no es una idea nueva y que el objetivo de los colegios agropecuarios va en esa dirección, también es cierto que la deserción de escuelas y colegios es sumamente alta. Entonces, de alguna manera el currículum actual no es atractivo para niños y jóvenes, y muchos de ellos apenas concluyen el sexto grado se dedican a trabajar como peones o en otras actividades no agrícolas (construcción, servicios, etc.). Las escuelas y los colegios de las zonas rurales deberían de ser fuentes de inspiración en el desarrollo rural de la zona donde están, y promover la formación y organización de jóvenes productores agrícolas. Herramientas para esto hay de sobra, lo que falta es la voluntad de aplicarlas. 


\section{Referencias}

Cartín S. \& Piszk, I. (1980). La Producción de Granos Básicos en Costa Rica, Instituciones Estatales y Fuerzas Sociales. Período de Diversificación Económica. In: Revista de Ciencias Sociales, $\mathrm{N}^{\circ} 19-20$. Universidad de Costa Rica.

Ellis, F. (1988). Peasant Economics, Farm Household and Agrarian Development. Second Edition. Cambridge University Press, UK. 309 p.

González Mejía, H. (1994). Desarrollo Agropecuario y Políticas Macroeconómicas en la Década del 80 en Costa Rica. En: Mora Al faro, J.; Oviedo Sánchez, O.; Fernández Alvarado, L.F. 1994. El Impacto de las Políticas Macroeconómicas en el Agro Costarricense. Facultad de Ciencias Sociales, Universidad Nacional. Editorial Universidad Nacional. 53-73 pp.

González Mejía, H. (1998). Agricultura y Competitividad en el Contexto de la Apertura Comercial. Paper presented in the Congress Costa Rica hacia el Siglo XXI. Balance de las Reformas Económicas para el Sector Agropecuario: 1983-1997 y Perspectivas. San José, Costa Rica.

Kaimowitz, D. (1992). Las Exportaciones Agrícolas No Tradicionales en América Central: su volumen y estructura. En: Exportaciones Agricolas No Tradicionales del Istmo Centroamericano ¿Promesa o Espejismo?, CADESCAPREALC (OIT), Panamá.

Kruseman, G.; Hengsdijk, H.; Ruben, R.; Roebeling, P.; Bade, J. (1997). Farm Household Modeling System for the Analysis of Sustainable Land Use and Food Security: Theoretical and Mathematical Description. Wageningen: AB-DLO/WAU, DLV Report N 7, 56 p.

La Gaceta. (1998). Decreto N²6639-Ministerio de Agricultura y Ganadería. Año $\mathrm{CXX}$, número 23. 1-4.

La Nación. 5 de octubre de 2002. EE.UU. ve difícil tema de agro de cara a TLC. P. $20 \mathrm{~A}$.

Masís, G. \& Rodríguez, C. (1994). La Inserción del Campesinado en un Proceso de Modemización no Incluyente. In: La Agricultura Campesina en Costa Rica: Alternativas y Desafios en la Transformación Productiva del Agro. Masís, G. \& Rodríguez, C. (eds). IDEAS. San José, Costa Rica. 
Mora Alfaro, J.; Oviedo Sánchez, O.; Fernández Alvarado, L.F. (1994), El Impacto de las Políticas Macroeconómicas en el Agro Costarricense. Facultad de Ciencias Sociales, Universidad Nacional. Editorial Universidad Nacional.

Mora Alfaro, J. (1994). Costa Rica: Políticas Agrícolas y Apertura Económica en los Años Noventa. Serie Política Económica N ${ }^{\circ} 14$. Maestría en Política Económica para Centro América y el Caribc, Universidad Nacional.

Noord, P.J. van der (1996) Globalisation and the European Disease. In: The Economist (144): 195-222.

Pomareda, C. (1996). Inistitutional Aspects of Sustainable Development. In: E. Castro \& G. Kruseman (eds) Policies for Sustainable Land Use in Costa Rica. Editorial Guayacán, CINTERPEDS-UNA/WAU, 41-54 pp.

Pomareda, C. (1998). Las Políticas Públicas y el Sector Agropecuario: Administración Figueres Olsen. San José, Costa Rica. MIDEPLAN y Asesorías Organizacionales.

Pomareda, C. (2000). Evolución y Perspectivas para la Agricultura de Costa Rica. Paper presented in the Congreso Nacional Agropecuario: Agricultura Costarricense al 2020. Organized by the Cámara Nacional de Agricultura y Agroindustria. San José, Costa Rica.

Sadoulet, E. \& de Janvry, A. (1995). Quantitative Development Policy Analysis. The Johns Hopkins University Press. Baltimore and London.

Secretaría Ejecutiva de Planificación Sectorial Agropecuaria (SEPSA). (1997). Políticas del Secior Agropecuario (Revisión y Ajuste). San José: Ministerio de Agricultura y Ganadería.

SecretaríaEjecutivade Planificación Sectorial Agropecuaria (SEPSA). (1999). Políticas para el Desarrollo Agropecuario y del Medio Rural Costarricense. San José: Ministerio de Agricultura y Ganadería. 